\title{
EXPERIENCIAS DE CÁTEDRA EN LAS CLASES DE FÍSICA DE PRIMER CURSO DE ESCUELAS TÉCNICAS
}

\author{
MESEGUER DUEÑAS, J.M. y MAS ESTELLÉS, J. \\ Departamento de Física Aplicada. Escuela Universitaria de Informática. Universidad \\ Politécnica de Valencia p.o. box 22012,46071 Valencia.
}

\begin{abstract}
SUMMARY
We have performed a set of lecture room experiences in a Physics course at the Escuela Universitaria de Informatica, of the Universidad Politécnica de Valencia; we have studied the effects produced on the students. This paper shows that we can prepare and perform this method easily, and also its low cost.

On the other hand, the general assessment of the students is positive, and it is particularly helpful to improve the motivation and the understanding of the concepts.
\end{abstract}

Durante el mes de septiembre de 1992, los autores asistimos al II Congreso Ybérico de Enseñanza de la Física, celebrado en Vila-Real (Portugal). Durante el mismo, tuvimos ocasión de asistir a la conferencia pronunciada por el profesor Rafael Márquez Delgado, del Departamento de Física de la Materia Condensada de la Universidad de Sevilla, que Ilevaba por título «El experimento de cátedra en la enseñanza de la Física». Esta conferencia reavivó en nosotros algunas ideas acerca de la realización de este tipo de experiencias en clase, que antes sólo habíamos puesto en práctica de forma aislada.

En el documento distribuido a propósito de la citada conferencia, el profesor Márquez (1992) aludía a los antecedentes, causas del declive en su utilización, ventajas pedagógicas y didácticas, y condiciones para su correcta realización, por lo que no abundaremos en estos aspectos.

No obstante, sí que haremos referencia a la utilidad que este tipo de experiencias presenta cuando se desarrollan en una clase magistral. En la mayor parte de los casos, el profesor se enfrenta en solitario ante los alumnos con la tiza en la mano, o a lo sumo con unas transparencias, como única herramienta de trabajo. Y esto sucede independientemente de que los grupos sean reducidos o numerosos, que la clase magistral se siga de tutorías, de prácticas de laboratorio, o que el método se base exclusivamente en la misma.
Hay materias que, en apoyo al trabajo del profesor en el aula, permiten utilizar como recursos las experiencias de cátedra. Éstas pueden ser fundamentales a la hora de motivar y orientar el aprendizaje del alumno, y la física sería un buen ejemplo de ello (Fig. 0). Este tipo de experiencias, típicas de las cátedras del siglo pasado y principios de éste (gabinetes de física), a pesar de su indudable utilidad, se han ido abandonando por falta de medios, imaginación y tiempo.

Figura 0




Por otro lado, Ias nuevas tecnologías también pueden auxiliar al profesor: la utilización de vídeos que muestren ejemplos, experiencias, aplicaciones... difíciles de llevar al aula; el ordenador, que mediante pantallas de cristal líquido, o proyectores de vídeo, permiten en el mismo momento en que se plantea una ley comprobar su validez, o ilustrar esquemas, dibujos, gráficos...

Nuestro trabajo lo hemos enfocado a la asignatura de Física de la EU de Informática, cuyos contenidos son: electromagnetismo, teoría de circuitos y semiconductores. El realismo a que nos lleva el tener 1.500 alumnos matriculados nos obliga a plantearnos el mejorar fundamentalmente las clases magistrales, ya que la introducción de otras metodologías, por ejemplo la basada en el trabajo tutorial, supone unas necesidades de recursos humanos y materiales que las hacen imposibles desde un punto de vista general. La idea de nuestro plan de trabajo fue:

- Analizar los 21 temas del programa obteniendo una lista de material de apoyo posible para cada uno de ellos: experiencias, vídeo, software.

- Programar la elaboración del trabajo, de forma que su realización tienda inicialmente a que cada capítulo o bloque disponga de un primer material. Así, se debía trabajar sobre los siguientes capítulos: introducción y concepto de la asignatura, electrostática, corriente continua, electromagnetismo, corriente alterna y teoría de circuitos y semiconductores.

El material elaborado es de aplicación inmediata y masiva, puesto que no interrumpe, sino que complementa, la metodología actualmente empleada. Además, se tenía la restricción de que como material debía utilizarse el ya disponible en el laboratorio de física, aunque posteriormente se recabó algún material adicional cedido por otros compañeros.

\section{TRABAJO REALIZADO DURANTE EL CURSO 1992-93}

Durante el curso 1992-93 hemos preparado y desarrollado algunas experiencias de cátedra que a continuación pasamos a detallar. En cada epígrafe indicamos el punto del programa en el que hemos planteado las experiencias. Entre paréntesis daremos cuenta de puntos concretos del programa de la asignatura a los que se van haciendo referencia.

\section{INTRODUCCIÓN INICIAL}

Mediante una disquetera antigua, grande, se puede comprobar, desmontándola en clase, la presencia de un circuito impreso (y hacer referencia a la teoría de circuitos) repleto de resistencias (corriente continua), condensadores (electrostática), transistores (física de semiconductores), chips..., dos motores eléctricos (fuerzas magnéticas) que controlan la posición del cabezal, una bobina (campo magnético) y el movimiento del disco (material magnético) en el que se puede escribir (iman- tación) o leer (inducción). Así hacemos un repaso conpleto al concepto de la asignatura y su programa.

\section{ELECTROSTÁTICA}

Con un generador de Van de Graaf, conseguimos cargar una esfera metálica a potenciales muy elevados. Esta carga nos sirve para poder realizar experiencias de electrostática:

Al colocar una peluca a la esfera, e ir cargándola, los pelos se erizan debido a las fuerzas de repulsión electrostática, dando un efecto espectacular que se convierte en una atractiva y divertida introduccion.

Separación de bolitas de saúco (aislantes) colocadas sobre la esfera (fuerzas de Coulomb).

No separación de las mismas bolitas de saúco situadas en el interior de un cilindro metálico (apantallamiento eléctrico, campo nulo en el interior de un conductor).

Movimiento de molinillo por efecto de «viento electrostáticon, y generación de descargas (rayos) continuos (efecto de puntas y campo de ruptura).

Mediante modelos transparentes -en los que se han situado, entre placas de metacrilato, electrodos de diferentes formas conectados al generador, y en su entorno semillas diminutas inmersas en un aceite- se pueden observar las líneas de campo eléctrico al producirse el alineamiento de las semillas por efecto electrostático. De esta forma podemos observar:

- Campo creado por carga puntual (un electrodo puntual, y el otro circular rodeando al primero). Se disponen las semillas de forma radial.

- Campo creado por esferas cargadas (electrodos circulares concéntricos). También se disponen en forma radial en el espacio entre los electrodos, y no se orientan en el interior del primero (campo nulo en el interior de un conductor).

- Electrodo plano. Las líneas de campo trazadas por las semillas son normales a su superficie en el punto de contacto con el electrodo (campo en las proximidades del conductor).

- Electrodos planos y paralelos. Líneas de campo normales a ambos en el espacio comprendido entre los dos (uniformidad del campo en el interior de un condensador plano).

Todo el material que acompaña al generador de Van de Graaff fue amablemente facilitado por nuestro compañero José Quilles.

\section{CONDENSADORES}

Se abrieron con herramientas (ensayo destructivo) distintos tipos de condensadores, de forma que se observase 
que en su mayor parte son planos, de gran superficie, con dieléctrico y con separación entre armadiras muy pequeña. Aparte de conocer cómo son, ratificamos ciertas hipótesis simplificativas que se realizan en la teoría sobre condensadores.

\section{MAGNETISMO}

Para introducir el magnetismo nos propusimos que la motivación fundamental fuesen las aplicaciones tecnológicas y también dar relevancia al papel que jugaron y juegan en el desarrollo económico y sociocultural de nuestro mundo. La energía mecánica, fácil de conseguir, se transformaba en energía eléctrica que mediante conductores se podía llevar donde nos interesase para transformarla en calor o de nuevo en energía mecánica. Los tres elementos en que nos basamos eran el generador de corriente, el motor y el transformador. Con una dinamo de bicicleta, un motor de coche de juguete y un transformador de radio, abriéndolos y desmontando sus elementos, hicimos hincapié en la sencillez de los tres, y en que el elemento común eran las bobinas. Las grandes instalaciones de generación de electricidad, los grandes motores y transformadores constaban de los mismos elementos y se basaban en los mismos principios.

Se realizaron las siguientes experiencias:

\section{Generador de corriente continua}

Con el fin de poner de manifiesto la gran importancia que tuvo para el posterior desarrollo del electromagnetismo la construcción de la primera pila, se generó una c.c. mediante una pieza de fruta o una raíz (manzana, limón, naranja, patata, etc.). Para ello, en una pieza de las mencionadas, se clavaron un trozo de cobre y un trozo de zinc, provocando la aparición de una d.d.p. entre ambos. Con el fin de obtener una d.d.p. mayor, se conectaron dos montajes de los anteriores en serie. Dado que aun así la corriente producida es muy débil, para detectarla se utilizó un pequefio reloj digital, de poco consumo. Los generadores mencionados permiten el funcionamiento del reloj durante una semana, aproximadamente.

Este montaje se encuentra disponible comercialmente.

\section{Fuerzas magnéticas}

Se vieron los efectos de un imán sobre distintos metales: llaves, clips... y un aro de aluminio que después se utilizará.

Con una brújula se vieron las fuerzas de atracción sobre imanes, utilizándose posteriormente la brújula como detector de campos magnéticos. Se establece la analogía de las fuerzas entre imanes con las fuerzas electrostáticas.

Una varilla metálica con un orificio en uno de sus extremos se suspende, a modo de péndulo, de un pequeño destorniliador sujeto, con una nuez, de un soporte de laboratorio. Del extremo inferior de la varilla, y deI extremo superior del soporte, mediante sendas pinzas cocodrilo, se obtienen dos bornes. Dichos bornes se conectan en serie con un solenoide de 1.200 espiras con un material ferromagnético en su interior, un amperímetro, y una fuente de c.c. La bobina se aproxima lo máximo posible a la varilla metálica, y al hacer circular una corriente de $1 \mathrm{~A}$ por el circuito, la varilla se desvía de su posición estable. Se demuestra que un conductor recorrido por una corriente dentro de un campo magnético, se ve sometido a una fuerza en la dirección y sentido del producto $\overrightarrow{\mathrm{L}} \mathrm{BB}$.

Se dispone de un motor eléctrico de pocas espiras, de dimensiones $5 \times 12 \mathrm{~cm}$ aproximadamente, que pueden girar dentro del campo magnético creado por un imán. Los anillos del motor se pueden colocar partidos o enteros, de forma que al girar se invierta, o no, la polaridad en las espiras. Si los anillos se colocan enteros, al hacer circular una pequefia corriente por el motor, las espiras giran hasta colocarse en el plano perpendicular al campo. Una espira recorrida por una corriente, se orienta en un campo magnético.

Si los anillos del motor se colocan de forma partida, las espiras giran ininterrumpidamente mientras se haga circular corrientes por ellas.

\section{Generación de campos magnéticos}

Con un conductor rectilíneo, un cable de cobre largo y estirado con las manos, por el que se hizo circular 1 amperio mediante un generador de c.c., se comprobó la aparición de fuerzas magnéticas sobre la brújula, que producían pequeñas desviaciones en su orientación, por lo que se deducía que las corrientes eléctricas creaban campo (Oersted). Como conductor rectilíneo se puede utilizar también un soporte de los habitualmente disponibles en cualquier laboratorio para sujetar pinzas y nueces. Es crítica la elección del soporte, ya que si es de un material ferromagnético con alta imantación remanente, al intentar repetir la experiencia por segunda vez, se constata la existencia de campo magnético en ausencia de corriente, lo cual puede causar confusión en el alumno.

Con una bobina de 600 espiras y mucha menos intensidađ, $0,5 \mathrm{~A}$, se conseguía desviar la brúfula de una forma mucho más fácil, y con una segunda bobina de 1.200 espiras, de una forma aún más intensa, por lo que se deducía que en la bobina se superponían los efectos de cada una de las corrientes de cada espira. Si se introducía en la bobina un cilindro de plástico no sucedía nada especial, pero si era de hierro, la intensidad de corriente necesaria era mucho menor y los efectos más apreciables, con lo que se comprobaba el comportamiento especial que tenían ciertos materiales que multiplicaban el efecto del campo magnético (ferromagnetismo).

\section{Fenómenos de inducción}

Con un imán largo y una bobina conectada a un amperfmetro, de tamaño grande para experiencias en aula, se 
observaban las corrientes inducidas al mover el imán dentro de la bobina.

\section{Corrientes de Foucault}

El anillo de aluminio se hacía levitar en el interior de un campo magnético de una bobina, creado por una corriente senoidal. Al finalizar la experiencia, los alumnos comprobaban que la temperatura del anillo era elevada, Io cual suponía efecto Joule o, lo que es lo mismo, que había circulado corriente por el anillo sin necesidad de generador.

Todas estas experiencias, aparte de su espectacularidad y de servir de introducción al capítulo, sirven individualmente para basarse en ellas a la hora de desarrollar cada uno de los puntos tratados.

\section{INDUCCIÓN}

En clase se hicieron las siguientes experiencias:

Con un imán targo y una bobina cortocircuitada sobre un amperímetro de aula, se observaban las corrientes inducidas al mover el imán. Las corrientes eran proporcionales a la velocidad a que se medía, y su sentido dependía del sentido del movimiento del imán, y del polo que se introdujese.

Con un generador formado por bobina de espiras rectangulares de $5 \times 12 \mathrm{~cm}$ aproximadamente situado en el interior del campo creado por un imán, haciéndola girar manualmente alrededor de su eje, y registrando en un osciloscopio la d.d.p. inducida entre sus extremos, se podía observar claramente el fenómeno de inducción, que la d.d.p. seguía una ley senoidal y que los valores de la amplitud aumentaban al hacerla girar más deprisa.

Con dos bobinas y un entrehierro cerrado, se alimentaba una de ellas con una d.d.p. senoidal mediante un generador de funciones. El osciloscopio registraba esta d.d.p. y la de la segunda bobina. Se podía apreciar el fenómeno de inducción y que la d.d.p. inducida era también senoidal de igual frecuencia. Jugando con bobinas de distinto número de espiras se podía comprobar cómo la relación entre las amplitudes de ambas d.d.p. senoidaies venía dada por la relación entre el número de espiras (fundamento del transformador).

Con las tres experiencias se recogían las tres formas fundamentales de generar inducción: movimiento en el elemento que genera el campo magnético, movimiento del circuito inducido y variación del campo al variar la corriente que lo crea.

El elemento común a las tres experiencias es únicamente la variación del flujo magnético, con lo que introducimos experimentalmente la ley de Faraday.

\section{MATERIALES MAGNÉTICOS}

La experiencia desarrollada pretende comprobar la validez de la teoría de los dominios para justificar los fenómenos de imantación y los ciclos de histéresis en los materiales ferromagnéticos.

Mediante dos modelos (Leybold 1986) que representan en el plano dos configuraciones cristalinas diferentes, y formados por gran número de brújulas, que modelizan ei momento magnético de cada átomo en la red, dispuestas en dos y tres direcciones respectivamente, se comproba$\mathrm{ba}$, en primer lugar, la existencia de direcciones de orientación preferente en los cristales; y, en segundo lugar, la aparición de zonas en las que todas las brújulas se orientaban en la misma dirección, los dominios.

$\mathrm{Al}$ aplicar un campo exterior mediante dos imanes se comprobaba cómo los dominios más favorecióos por la dirección del campo crecían de tamaño, hasta que, al estar todas las brúfjulas en estas direcciones, empezaban a girar en la dirección del campo. Al final se llegaba a una situación de máxima imantación con todas las brújulas orientadas en la misma dirección, la saturación, que no podía ser superada (imantación de saturación). Al eliminar el campo retirando los imanes se comprobaba que volvían a aparecer los dominios, pero los que habian sido más favorecidos por el campo tenían claramente un tamaño superior, por lo que el cristal no estaba desimantado (imantación remanente). Al aplicar el campo en sentido contrario se volvía, con el mismo proceso, a la saturación, y eliminando el campo se obtenía de nuevo una imantación remanente. Aplicanđo de nuevo el campo y liegando a la saturación inicial, se cerraba el ciclo de histéresis del modelo.

Con ambos modelos se obtenfan resultados semejantes, pudiendo comprobar el efecto que tiene el hecho de que el campo se aplique en una de las direcciones preferentes (mayor imantación remanente), y el hecho de tener menor número de direcciones preferentes (mayor imantación remanente también).

Este equipo se encuentra disponible comercialmente.

\section{INTRODUCCIÓN A CORRIENTE ALTERNA}

Buena parte de la totalidad de las prácticas de laboratorio de la asignatura son de corriente alterna, por to que los alumnos irán descubriendo aplicaciones y posibilidades. En clase desarrollamos un ejemplo sobre las posibilidades matemáticas de las funciones senoidales, que son los desarrollos en serie de Fourier.

El modelo se presenta en clase mediante pantalla de cristal líquido conectada a ordenador. El problema es la comunicación entre ordenadores mediante conductores. En este punto del programa, los alumnos saben que los conductores tienen resistencia, capacidad y autoinducción, y se han calculado en lecciones anteriores en función de su geometría y características materiales. Esto supone, como verán en la práctica correspondiente, 
que funcionan como filtros, eliminando señales de frecuencias que estén fuera de determinados rangos. El problema es determinar hasta qué punto este efecto de filtrado nos modifica las señales lógicas (ceros y unos) que se trasmiten por los conductores. El programa calcula la suma de los términos del desarrollo en serie de Fourier permitidos por nuestro conductor, y representa gráficamente la señal que sale del primer ordenador y la que le llega al último. Se puede representar en pantallas sucesivas el efecto de ir alargando el conductor, y la pérdida de información que supone.

\section{SEMICONDUCTORES}

Mediante un chip se recuerda la importancia que tienen los semiconductores en toda la tecnología moderna. Con una oblea de silicio, de las utilizadas en la industria de semiconductores, se señala que la tecnología que se emplea en sus aplicaciones es muy sofisticada, por la necesidad de controlar la pureza de los materiales empleados y por la exactitud que se debe conseguir en las miniaturizaciones. A pesar de ello, es una tecnología muy desarrollada.

Para plantear las diferencias cualitativas y cuantitativas entre conductores y semiconductores se utiliza una resistencia de platino (un sensor de temperatura Pt 100) y un semiconductor (un diodo al que se le ha quitado la protección). Mediante un polímetro se mide su resistencia, comprobando que la del conductor es bastante menor a pesar de que la sección del conductor es mucho menor que la del semiconductor y su longitud mucho mayor. El diodo, aun estando en polarización directa, nos dará unos valores de resistencia apreciables debido a que la d.d.p. empleada por los polímetros para medir las resistencias está dentro del codo inicial de la curva característica del mismo.

Después, colocándola en la mano se comprueba cómo la resistencia del conductor aumenta al subir ligeramente su temperatura, comportamiento que está conforme con el modelo de conductor que conocen los alumnos. Seguidamente, por medio de un secador de pelo se calienta el semiconductor, obteniendo un resultado opuesto: la resistencia disminuye, y de una forma relativamente más importante que el aumento del conductor.

A continuación, con una fotoresistencia de semiconductor se comprueba el mismo efecto de disminución de la resistencia al iluminarlo, aumentando progresivamente la resistencia al oscurecer el semiconductor. Se les explica que ambas experiencias suponen un aporte de energía al semiconductor, en el segundo caso la energía de los fotones, y que en ambos casos la conductividad aumenta. Este comportamiento no se puede justificar mediante el modelo de conductor (nube de electrones libres), y plantea la necesidad de un modelo de conducción diferente para los semiconductores.

Por otra parte, y con el fin de ilustrar otra aplicación de los semiconductores, se les mostró una pequeña placa compuesta de células fotovoltaicas. Al exponer la placa a un rayo luminoso, se genera una d.d.p. entre los extremos de la placa, d.d.p. que es tanto mayor cuanto más intensa es la luz incidente. Al alimentar con estas células un pequeño motor eléctrico, por ejemplo, se pone de manifiesto el efecto deseado.

Por último, con un djodo, presentado como componente electrónico hecho de material semiconductor al que se le han introducido impurezas de otro material con distribución no uniforme, se comprueba cómo la resistencia es diferente según se conecte a los bornes del polímetro: alta en un sentido, muy baja en el otro. Evidentemente este comportamiento no es justificable mediante la ley de Ohm, y abre la puerta a aplicaciones nuevas. Con un generador de funciones se suministra una d.d.p. senoidal de frecuencia muy baja a la resistencia de platino y al diodo, midiendo la intensidad de corriente que circula por ellos mediante un amperímetro. Se puede comprobar cómo la intensidad en el conductor es también senoidal, mientras que en el diodo sólo hay corrientes en un sentido, no dejando circular la corriente en sentido contrario.

\section{EFECTO DE LAS EXPERIENCIAS REALIZADAS}

Con el propósito de estudiar el efecto que sobre los alumnos ha tenido el trabajo realizado, se ha pasado a un grupo de ellos una encuesta de contestación objetiva en la que se les pide una valoración sobre distintos aspectos de las experiencias realizadas y la valoración global de cada una de ellas.

Se plantearon 12 afirmaciones, a las que debían contestar según su opinión:

1. En total desacuerdo.

2. Más bien en desacuerdo.

3. Indiferente

4. Más bien de acuerdo.

5. Totalmente de acuerdo.

Las afirmaciones de la encuesta eran las siguientes:

En general, las experiencias de cátedra:

1. Ayưan à entender la teoría.

2. Despiertan el interés sobre el tema.

3. Se comprendían con facilidad.

4. No tenían complicaciones técnicas excesivas (sofisticación).

5. Rompen la monotonía de la clase.

6. Hacen más divertida la clase.

7. El profesor las ha realizado en clase correctamente.

8. El profesor las tenía bien preparadas.

9. El profesor se apoyó en ellas para desarrollar la teoría.

10. Es adecuada su utilización en asignaturas como la física.

11. Podrían utilizarse con más frecuenciâ.

12. Globalmente, las experiencias realizadas me parecen adecuadas. 
La encuesta se hizo en cuatro grupos: dos, de mañana y tarde, que tenían al mismo profesor (profesor 1), y otros dos que correspondían a otro profesor (profesor 2), con el fin de estudiar posibles deficiencias de la misma y también el posible efecto de la intervención del profesor. El número total de alumnos era 66 del profesor 1 y $98 \mathrm{del}$ profesor 2 , todos ellos con asistencia habitual a clase a lo largo de todo el curso. La encuesta se realizó en la penúltima semana del curso, entre los asistentes a clase. Los resultados globales son los siguientes:

\begin{tabular}{|c|c|r|r|r|r|r|r|r|}
\hline $\mathrm{A}$ & 1 & 2 & 3 & 4 & 5 & $\mathrm{n}$ & $\mathrm{X}$ & $\mathrm{S}$ \\
\hline $\mathrm{I}$ & & 3 & 20 & 79 & 62 & 164 & 4,2 & 0,7 \\
2 & & 2 & 17 & 68 & 77 & 164 & 4,3 & 0,7 \\
3 & & 6 & 24 & 99 & 34 & 163 & 4,0 & 0,7 \\
4 & & 6 & 33 & 84 & 38 & 161 & 4,0 & 0,8 \\
5 & 3 & 6 & 4 & 27 & 123 & 163 & 4,6 & 0,8 \\
6 & & 2 & 7 & 40 & 114 & 163 & 4,6 & 0,6 \\
7 & & 1 & 7 & 67 & 89 & 164 & 4,5 & 0,6 \\
8 & & & 8 & 63 & 91 & 162 & 4,5 & 0,6 \\
9 & 1 & 4 & 29 & 77 & 52 & 163 & 4,1 & 0,8 \\
10 & & & 4 & 55 & 105 & 164 & 4,6 & 0,5 \\
11 & & 5 & 17 & 62 & 80 & 164 & 4,3 & 0,8 \\
12 & & & 9 & 71 & 84 & 164 & 4,5 & 0,6 \\
\hline
\end{tabular}

A= nứnero de cada afimación $\mathrm{n}=$ número de contestaciones

$\mathrm{X}=$ valor medio de las respuestas

$S=$ desviación tipica

De los resultados se pudieron observar las pequeñas diferencias entre los dos grupos de cada profesor, y entre los resultados de los dos profesores (en todos los casos iguales o inferiores a una décima de punto respecto de la media del conjunto total de alumnos), lo que avala la consistencia del test, y la objetividad de los resultados. Por otro lado, la desviación típica es aceptable, lo que nos indica poca dispersión en las contestaciones (Fig. 1). Dando valores a cada contestación de 1 a 5, la međia para todas las afirmaciones está por encima de 4 (Fig. 2), es decir, entre la contestación «más bien de acuerdo» y la contestación «totalmente de acuerdo». Sólo las afirmaciones 3 y 4 obtienen dicha puntuación $(4,0)$, haciendo referencia a la motivación y la sofísticación de las experiencias, to cual supone una valoración positiva de todos los aspectos relacionados con la experiencia y tratados en la encuesta. Se deben destacar los resultados de las afimaciones siguientes: 5 , «rompen la monotonía de clase» $(4,6) ; 6$, «hacen más divertida la clase» $(4,6)$; 7 , «el profesor las ha realizado correctamente» $(4,5) ; 8$, «el profesor las tenía bien preparadas» $(4,5) ; 10$, «es adecuada su utilización en asignaturas como la física» $(4,6)$; y 12 , «globalmente, las experiencias realizadas me parecen adecuadas» $(4,5)$. Las dos primeras están relacionadas con la amenidad de las clases, las dos segundas sobre el trabajo del profesor, y las dos títimas sobre la adecuación de las experiencias. En los tres casos la valoración es positiva, coincidiendo además con las respuestas de menor dispersión de resultados.
Figura 1

Distribución de las respuestas para cada afirmación.



Figura 2

Valor promedio obtenido por cada afirmacion (máximo 5).

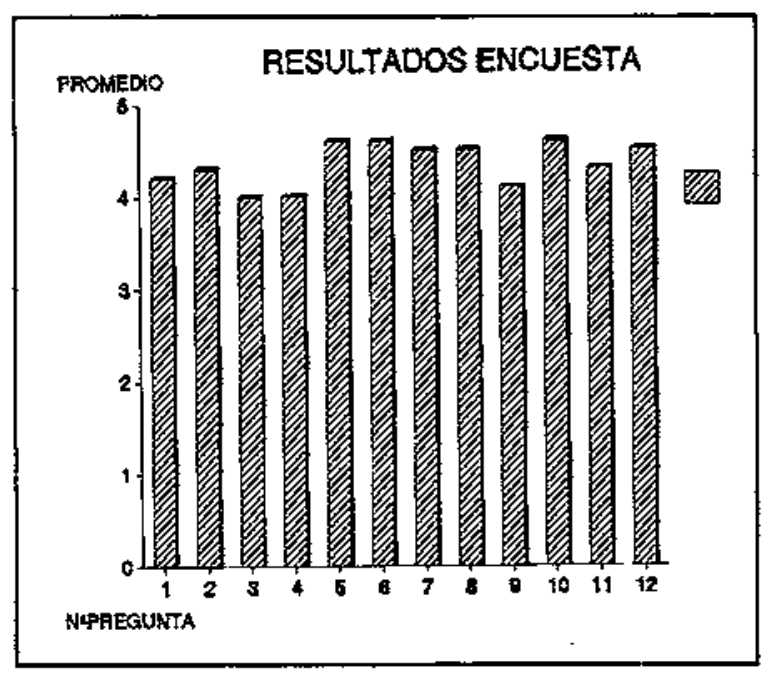

La valoración de cada experiencia, en la que el alumno puntuaba de 0 a 10 , se realizó en los grupos del profesor 1 (grupos A y H), con un total de 66 alumnos. Se ha seguido el siguiente orden:

1. Introducción (disquetera).

2. Generador Van Der Grafft (electrostática).

3. Introducción al magnetismo.

4. Inducción.

5. Materiales magnéticos.

6. Introducción C.A. (Fourier).

7. Semiconductores. 
Los resultados por grupo y globales son los siguientes:

\begin{tabular}{|c|cc|cc|c|c|c|}
\hline \multicolumn{9}{|c}{ GRUPO A } & \multicolumn{3}{c|}{ GRUPO H } & \multicolumn{3}{c|}{ TOTAL } \\
Ex & Xa & na & Xh & nh & X & S & n \\
\hline 1 & 6,8 & 22 & 6,5 & 19 & 6,7 & 1,4 & 41 \\
2 & 8,0 & 23 & 7,5 & 22 & 7,8 & 1,3 & 45 \\
3 & 8,1 & 23 & 7,6 & 20 & 7,9 & 0,9 & 43 \\
4 & 7,7 & 23 & 8,1 & 20 & 7,9 & 1,1 & 43 \\
5 & 7,7 & 20 & 7,7 & 14 & 7,7 & 1,2 & 34 \\
6 & 7,6 & 20 & 7,6 & 16 & 7,6 & 1,3 & 36 \\
7 & 8,3 & 26 & 8,2 & 21 & 8,2 & 1,1 & 47 \\
\hline
\end{tabular}

$\mathrm{Xa}$ y $\mathrm{Xh}=$ valoración media en los grupos $\mathrm{A}$ y $\mathrm{H}$

na y nh= número de respuestas en los grupos $\mathrm{A}$ y $\mathrm{H}$

$X=$ valor medio total

$\mathrm{S}=$ desviación típica

n= número de respuestas total

Distribución de resultados para cada experiencia:

\begin{tabular}{|c|c|c|c|c|c|c|c|c|}
\hline \multicolumn{10}{|c|}{ PUNTUACIONES np (\%) } \\
\cline { 2 - 8 } Ex & 4 & 5 & 6 & 7 & 8 & 9 & 10 & $\begin{array}{c}(\%) \\
>=7\end{array}$ \\
\hline 1 & $2(5)$ & $6(15)$ & $10(24)$ & $13(32)$ & $5(12)$ & $3(7)$ & $2(5)$ & 56 \\
2 & & $3(7)$ & $5(11)$ & $9(20)$ & $13(29)$ & $12(26)$ & $3(7)$ & 82 \\
3 & & & $3(7)$ & $10(23)$ & $21(49)$ & $7(16)$ & $2(5)$ & 93 \\
4 & & & $6(14)$ & $7(16)$ & $19(44)$ & $7(16)$ & $4(10)$ & 86 \\
5 & & $1(3)$ & $4(12)$ & $10(29)$ & $11(32)$ & $5(15)$ & $3(9)$ & 85 \\
6 & $1(3)$ & $1(3)$ & $5(14)$ & $10(28)$ & $10(28)$ & $7(18)$ & $2(6)$ & 80 \\
7 & & $2(4)$ & $1(2)$ & $8(17)$ & $18(38)$ & $10(21)$ & $8(17)$ & 93 \\
& & & & & & & \\
\hline
\end{tabular}

$\mathrm{Ex}=$ número de la experiencia de cátedra

(\%) $>=7=$ tanto por ciento de valoraciones iguales o superiores a 7 np= número de respuestas de una puntuación determinada

En este caso, las calificaciones medias Ilegan a diferenciarse como máximo en 0,5 puntos entre los dos grupos. En todos los casos en que hay una diferencia de este orden entre los dos grupos coincide el de menor valoración con el grupo en el que la experiencia se realizó en primer lugar, por tanto podría interpretarse como atribuible al profesor: la experiencia hace que se corrijan errores que se cometen en la primera ocasión.

Globalmente las desviaciones típicas son aceptables; las puntuaciones, medias elevadas, y la distribución de las puntuaciones, coherente (Fig. 3 y 4). Excepto en la primera experiencia, que obtiene una valoración media de 6,7 puntos, el resto obtiene valoraciones iguales o superiores a 7 en un porcentaje superior al $80 \%$, destacando la tercera (introducción al magnetismo) y la séptima (semiconductores) con el 93\% (Fig. 5).
Figura 3

Calificación media obtenida por cada experiencia (máximo 10).

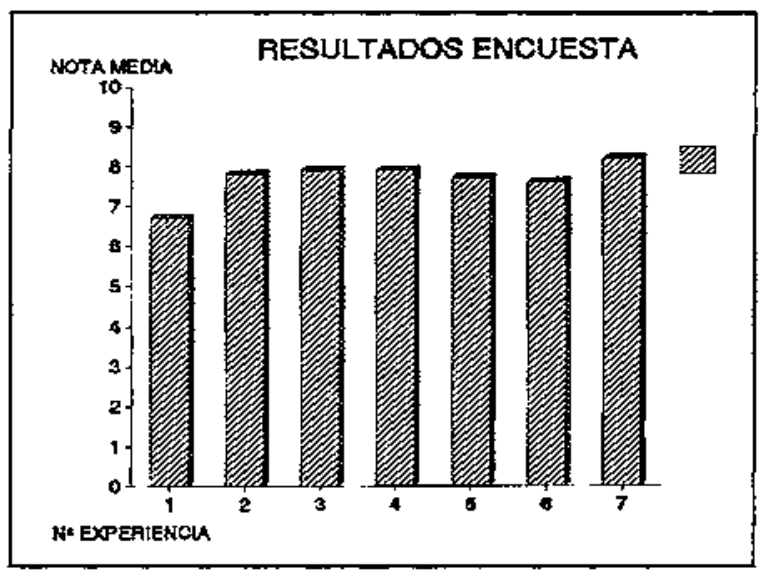

Figura 4

Distribución de las calificaciones para cada experiencia.
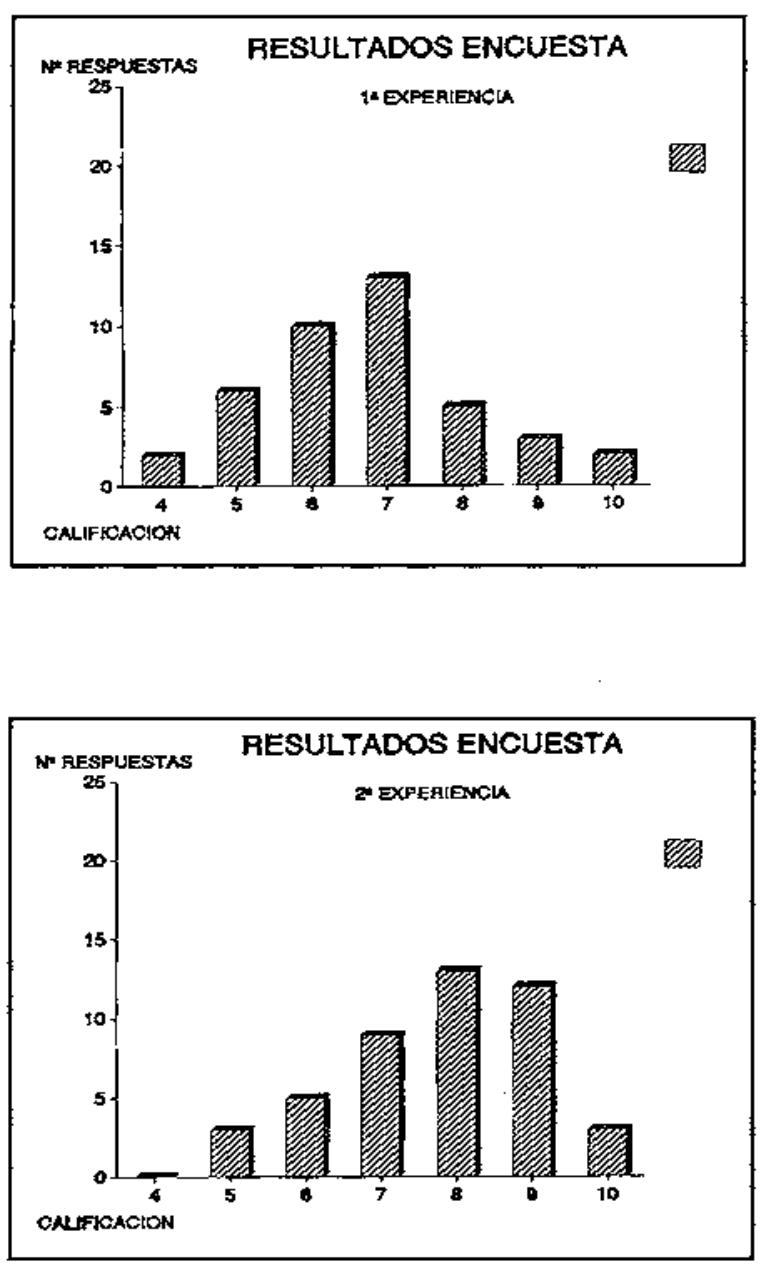

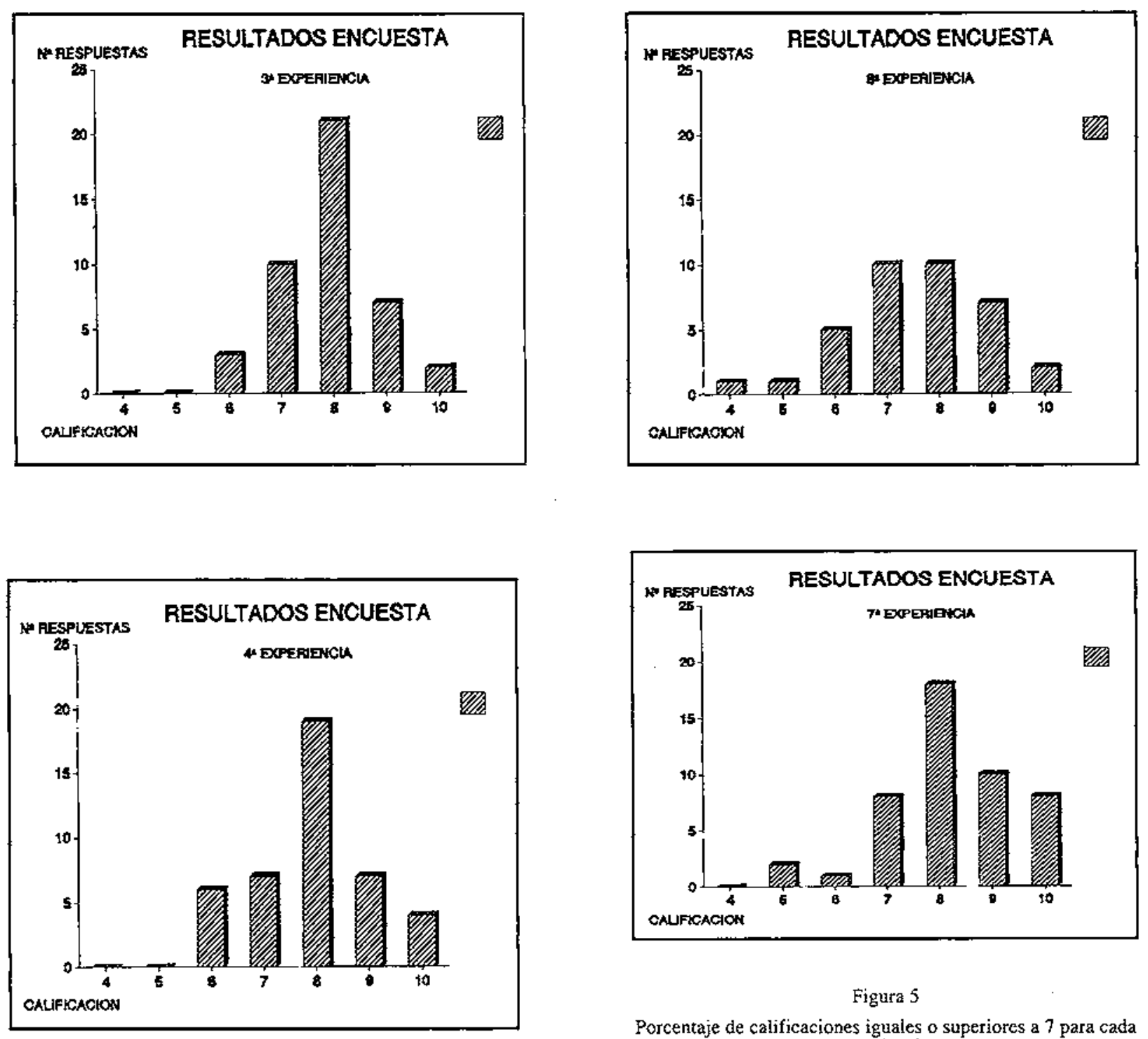

Figura 5

Porcentaje de calificaciones iguales o superiores a 7 para cada experiencia.

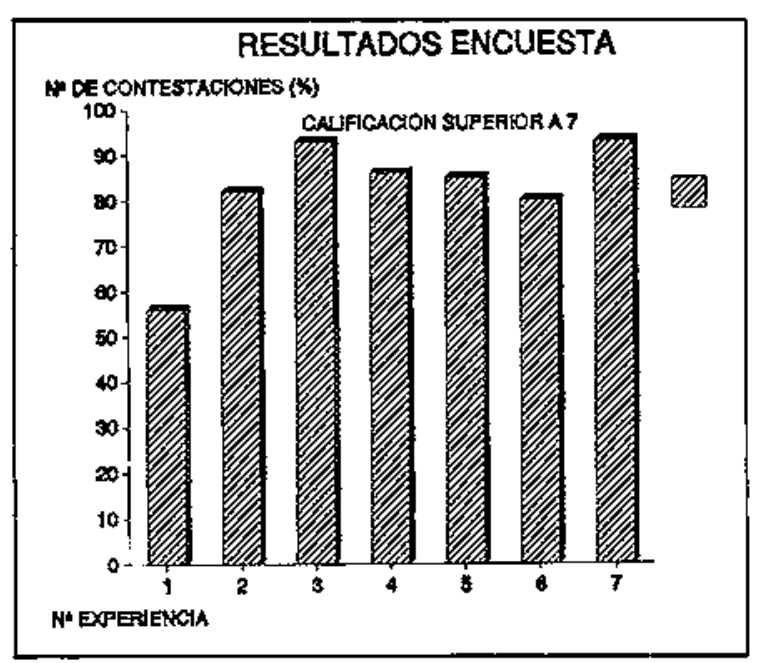


También es de destacar que de los 66 alumnos a los que se realizó la encuesta, en todos los casos más de 20 , una tercera parte no sabe cómo valorar las experiencias, a pesar de que las han presenciado todas. Este dato podría cuestionar la validez absoluta de los resultados de estas calificaciones, aunque de todas formas pensamos que son suficientemente significativos. Sería interesante estudiar la capacidad que tienen los alumnos no sólo de valorar el trabajo de otros, sino de valorar su propio trabajo, su sentido crítico y autocrítico.

\section{DISCUSIÓN}

El trabajo realizado nos ha hecho constatar algo que por otro lado parece evidente: no todos los temas se prestan con igual facilidad a la realización de experiencias. Para nuestra asignatura, por sus contenidos, es bastante fácil, pero a pesar de ello determinadas experiencias fueron complejas de desarrollar. Por ejemplo, en electrostática tuvimos la suerte de encontrar un generador de Van Der Graaf, ya que en caso contrario no hubiese resultado tan sencillo elaborar una experiencia tan completa. Por contra, todas las de magnetismo surgían sin esfuerzo, dada la gran cantidad de aplicaciones tecnológicas sencillas que existen. Por lo tanto, la generalización del método a otros casos dependerá đe sus contenidos particulares y de los recursos de que se disponga.

El tiempo dedicađo a la realización de las experiencias en ningún caso ha sido superior a la media hora, por lo que difícilmente se puede objetar que suponen un retraso en la marcha normal del curso, más aún cuando los đesarrollos teóricos se vayan respaldando en las experiencias, ya que será más fácil y breve recordarles algo que han observado experimentalmente que describirles los resultados de lo que no ven.

No se buscaba mejorar de forma significativa los resultados globales de los alumnos, y sería difícil, o como mínimo muy laborioso, el estudio de dichos efectos sobre el rendimiento de los alumnos. El propósito era más bien facilitar la comprensión de conceptos; y la respuesta a la primera afirmación de la encuesta («facilitan la comprensión de la teoría», con una puntuación promedio de 4,2 ) apoya el éxito de dicha intención. Por otro lado, de los resultados de la encuesta se deduce que el efecto más evidente es de tipo motivador, despertando el interés por los temas y haciendo más atractivas las clases.

Todas las experiencias realizadas son sencillas en su ejecución en clase; no se precisa habilidad especial por parte del profesor. Los instrumentos utilizados son tos que los alumnos conocen de las prácticas de laboratorio, y de las respuestas a la encuesta se deduce que la mayoría no encuentra que las experiencias tengan una complejidad excesiva que dificulte su comprensión, aunque sí existe un número significativo de respuestas en sentido contrario (más adelante pasaremos a comentarlo). En algún caso han aparecido dificultades no previstas: un conductor estropeado, un fusible fundido... De cara a los alumnos, estos problemas del profesor se han acogido con naturalidad, pero evidentemente el profesor debe confiar en su experiencia para saber reconducir tales situaciones, y compensarlas con experiencias equivalentes improvisadas sobre la marcha (por ejemplo: en una de las veces que se realizó la experiencia de semiconductores, al someter el diodo a una d.d.p. senoidal, el cable coaxial estaba estropeado y no se registraba respuesta. Mediante una pila que sustituía el generador, $y$ cambiando la polaridad, los alumnos podían observar que el diodo conducía con una polaridad de la pila, y con la otra no. No se pudo ver su efecto rectificador sobre corrientes aiternas, pero sí se pudo comprobar la diferente resistencia con la polaridad).

Para el trabajo realizado, el material que se utilizó era del disponible en el laboratorio de prácticas de nuestra asignatura, y material desechado de los laboratorios de prácticas de otras asignaturas del departamento, por lo que no se ha precisado invertir recursos económicos. La lista de material es la siguiente:

1. Introducción inicial:

Disquetera de desecho y disco magnético.

\section{Electrostática:}

Generador de Van Der Graaf.

Kit de demostración de campos eléctricos.

Peluca.

Diversos cables conductores.

3. Condensadores:

Diversos condensadores para destruir.

4. Corriente continua:

Equipo para generador de frutas (generador de corriente continua que utiliza como electrolito pulpa de fruta).

5. Magnetismo:

Dos imanes.

Una aguja imantada (brújula).

Soporte de laboratorio.

Generador de c.c.

Amperímetro de pantalla grande.

Bobina de 600 espiras.

Bobina de 1200 espiras.

Trozo de plástico encajable en las bobinas.

Trozos de material ferromagnético encajables en las bobinas.

Varios cables conductores.

Alambre conductor rígido.

Nuez para soporte laboratorio.

Motor/generador eléctrico con anillos partidos/sin partir.

Anillo de aluminio.

Controlador de potencia de c.a. (220 V.)

Generador de c.a.

Osciloscopio.

Kit de materiales ferromagnéticos.

Destornillador.

6. Semiconductores:

Termoresistencia.

Fotoresistencia. 
Diodo.

Placa de generadores fotovoltaicos.

Se puede constatar lo limitado de las necesidades y el aprovechamiento que se puede obtener de ellos.

No podemos plantear el problema del tiempo dedicado en la preparación de las experiencias de una forma general. Aunque el guión se puede plantear con relativa facilidad, puesto que debe adaptarse a las necesidades de los contenidos de la asignatura, el crear, idear, experiencias sencillas no es tan inmediato. Posteriormente, et llevar la idea a la práctica y superar las dificultades experimentales que van surgiendo también precisa una gran dedicación. Hay ocasiones en las que aparecen problemas a los que no se encuentra una solución rápida, aunque ésta al final sea sencilla (ejemplo: el correcto funcionamiento deI generador electrostático depende de la posición de las escobillas y del calentamiento de la cinta aislante, que se consigue simplemente manteniéndola en funcionamiento y dejando que rocen durante un cierto tiempo). De todas formas, este trabajo resulta atractivo y gratificante por los resultados, y la única (?) dificultad para el profesor es la general falta de tiempo. Una posible opción de cara a la preparación de las experiencias sería contar con los alumnos. Se podría atraer a alumnos, en general poco motivados por colaborar en asignaturas básicas, mediante becas de colaboración o a través de "proyectos de final de carrera», en los casos en que éstos sean posibles. De esta forma no sólo se tendría ayuda en el trabajo, sino que se dispondría de alguien que nos daría una visión inmediata de las virtudes y defectos de las experiencias desde el punto de vista del alumno.

Algo que parece evidente es que para rentabilizar el esfuerzo es necesario apoyarse en las experiencias magistrales a Ia hora de desarrollar los conceptos teóricos de la asignatura. De alguna forma, éstos son los que condicionan cuáles son las experiencias a realizar, y uno de los propósitos fundamentales es ayudar en su comprensión. Será, por lo tanto, conveniente que hagamos referencia lo más continuamente posible a las experiencias llevadas a cabo, utilizando dibujos que puedan recordárselas, o incluso volviendo a llevar a clase algún elemento característico de ellas, como puede ser una bobina, un diodo, un imán...

El modelo de aula más habitual, de forma rectangular y plana, con tarima en el frente, no nos parece el más adecuado para el seguimiento de experiencias de cátedra. Aulas de estilo anfiteatro, en las que la distancia del profesor al alumno se minimiza, serían las idóneas. Si además dispusiesen de medios audiovisuales (cámaras de vídeo, monitores...), la situación sería ideal. Evidentemente éste es un problema de difícil solución en grupos numerosos, y sólo se puede remediar parcialmente utilizando aparatos especiales para experiencias de clase, de tamaño superior al normal y mejor visualización, que todas las marcas comerciales de instrumentación de laboratorio docente tienen en sus catálogos. Esto puede ocasionar que el costo de las experiencias se haga mayor, aunque son inversiones que se podrían rentabilizar con rapidez compartiéndose entre varios profesores. Noso- tros hemos utilizado muitímetros de este tipo con excelente resultado, pero teníamos ciertas dificultades con otros elementos, por ejemplo con los osciloscopios, con los pequeños modelos de motores y generadores eléctri$\cos , y$ con otras experiencias de induccion. De todas formas, siempre tendremos que utilizar algunos elementos de tamaño reducido, tales como componentes de circuitos electrónicos, cuya visualización presentará problemas en cualquier caso.

Las dificultades de comprensión de las experiencias debidas a la posible complejidad de los montajes experimentales es un aspecto importante a considerar. Aunque de los resuitados de la encuesta se deduce que en general no es un problema, no se debe olvidar que un $18 \%$ de los alumnos en la afirmación tercera, y un $24 \%$ en la cuarta, consideran que les resultaba «indiferente» o se mostraban «más bien en desacuerdo». Indudablemente se trata de uno de los aspectos fundamentales a la hora de diseñar la experiencia de cátedra: conseguir un equilibrio entre la simplicidad de la experiencia y la complejidad abstracta de los conceptos que se desee ilustrar con ella. En numerosas ocasiones resulta difícil ponerse en la piel del alumno para analizar estos aspectos, y es necesario hacer ensayos en clase para comprobar los resultados, realimentándose con el efecto que cause sobre los alumnos. También se ha de señalar que en determinadas ocasiones sucede todo lo contrario: experiencias en las que se esperan problemas resultan totalmente clarificadoras para los alumno. Por ejemplo la de materiales ferromagnéticos: en todas las ocasiones en que se han empleado aspectos tan complejos como la imantación, ciclos de histéresis y teoría de los dominios quedan absolutamente claros en un tiempo muy breve y con una experiencia sencilla.

En las carreras de tipo tecnológico es conveniente relacionar to antes posible las asignaturas básicas con las aplicaciones. En nuestro caso, buen número de las experiencias lo han pretendido (disqueteras, motores, generadores, transformadores, comunicación entre ordenadores...). Apoyándonos en ellas podemos remarcar la sencillez de la mayor parte, consecuencia inmediata de la sencillez de las leyes f́́sicas en las que se basan.

Relacionado con lo anterior, consideramos como un aspecto importante del método el llevar a clase problemas reales. En muchos casos, para el alumno, la física se convierte en una asignatura de contenidos abstractos, en la que se plantean problemas ideales $s i n$ aplicación real, cuando es justamente lo contrario. Comprobar en clase que las leyes se cumplen, que se pueden plantear de forma teórica problemas sencillos reales, que determinadas simplificaciones en los problemas no introducen errores importantes y que las soluciones teoricas coinciden con los resultados experimentales puede ayudar a sacarles de ese error.

El hecho que el profesor tenga el papel fundamental en la ejecución de las experiencias no debe impedir que esté continuamente pendiente de las reacciones de los alumnos. Aunque el nuevo papel del profesor como experimentador refuerce su rol en el aula, no debemos olvidar que los alumnos aprenden más fácilmente lo que practi- 
can, y que, por lo tanto, la situación ideal sería que ellos mismos diseñasen y realizasen las experiencias. De alguna forma sería adecuado tener estos aspectos en consideración pidiendo la colaboración de alumnos en la ejecución de las experiencias.

Por último hemos de constatar la falta de bibliografía actual sobre el tema, particularmente para el caso de la física. Sí que se encuentran textos antiguos y bibliografía sobre prácticas de Iaboratorio, pero difícilmente sobre experiencias que sean útijes para realizar en clase.

\section{CONCLUSIONES}

La conclusión más importante que se puede obtener es la positiva valoración global que los alumnos hacen de las experiencias de cátedra realizadas. Otras consideraciones a tener en cuenta son las siguientes:

En primer lugar, y en lo gue a la actividad del profesor respecta, hay que resaltar la facilidad en su realizacion; para ello sólo es necesario un poco de imaginación. Esta facilidad choca con la necesaria disponibilidad de tiempo.

En segundo Iugar, este tipo de experiencias abre la posibilidad de utilizarlas como referencia y apoyo, de forma continuada, durante el desarrollo de las clases teóricas.
En lo que a los alumnos respecta, este tipo de experiencias supone una motivación para el seguimiento del temario.

Respecto al material utilizado, destacamos los pocos recursos materiales necesarios, to que posibilita y aconseja la utilización de las experiencias de cátedra.

La utilización de un material excesivamente complicado o sofísticado, si no es convenientemente explicado al alumno, puede provocar en éste la incomprensión de la experiencia.

Finalmente hay que resaltar el problema de la falta de visibilidad desde las últimas filas de la clase. Este aspecto debería ser solucionado o bien reduciendo el número de alumnos por grupo, o bien mediante monitores de televisión distribuidos por el aula.

\section{AGRADECIMIENTOS}

A José Quiles, por conseguirnos equipos y darnos ideas. A Amparo Estrada, por su dibujo.

\section{NOTA}

Este trabajo se realizo con medios materiales y humanos de la Unidad Docente de la EU de Informática del Departamento de Física Aplicada de la Universidad Politécnica de Valencia.

\section{REFERENCIAS BIBLIOGRÁFICAS}

ARONS, A.B., 1990. A Guide to Introductory Physics Teaching. (Ed. J. Willey and sons).

LEYBOLD, 1986. General Catalogue of Physics Experiments, p. 251 .
MÁRQUEZ, R., 1992. «El experimentode cátedraen la enseñanza de la Física». Libro de Actas del II Encuentro lbérico para la Enseñanza de la Física, Vita-Real (Portugal).

ZHDANOV, L., 1980. Manual de Física. (MIR: Moscú). 